\title{
EDITORIAL
}

\section{Regulation of energy balance-classical concepts and novel insights}

European Journal of Clinical Nutrition (2017) 71, 293; doi:10.1038/ejcn.2016.257

Progress in scientific knowledge on body weight regulation seems to be outpaced by the obesity epidemic. Nutritional advice for weight management is characterized by a confusing variety and partly conflicting content of messages that all in a way claim to be scientifically proven. Unequivocal conclusions from different studies may be due to discrepant levels of evidence (for example, observational vs intervention studies) or heterogeneity of the phenotype studied (for example, healthy lean vs obese patients). The biggest problem is however that the regulation of energy balance can only be studied when energy balance is measured accurately. This obvious prerequisite today still remains a major challenge to research on body weight regulation.

Body composition analysis, doubly labelled water and movement sensors can be used to monitor and explain long-term changes in energy balance. Short-term regulation of energy balance is however not captured by these methods due to methodological limitations. Although overweight and obesity are commonly explained as a consequence of a chronically positive energy balance, this interpretation is an oversimplification that bears the risk of missing the underlying mechanisms contributing to long-term weight gain. In reality, energy intake and expenditure vary from day to day and even within a day, for example, the balance is negative overnight and positive at daytime, leading to a considerable fluctuation in energy balance. Brief periods where energy intake far exceeds energy expenditure last from one meal to several days and regularly occur over the weekend, on holidays, at periods of celebration or during vacations. Gradual weight gain therefore more likely results from repeated short periods of positive energy balance that are inadequately compensated for.

Short-term regulation of energy balance can be studied on a daily basis using controlled feeding and indirect calorimetry in combination with nitrogen balance. Respiratory chambers have long been used in nutritional sciences to measure energy and macronutrient balances in order to gain insights into the regulation of body weight and partitioning of fat and lean mass. Today, in the era of 'omics' and molecular science, traditional methods of integrative physiology may appear laborious, oldfashioned and partly outdated. Yet, they still remain the clue for understanding the functioning of the organism as a whole. Although 'big data' approaches are appealing to modern scientists, many of the generated hypotheses fail to be replicated and verified by human intervention studies.
Today, comprehensive expertise on ever-advancing methods is increasingly outsourced to specialized methodological platforms that act as service providers to joint research projects. Scientific projects that can afford these platforms have become larger in number and involve thousands of participants. On the other hand, the drawback of small numbers in basic physiology studies can partly be overcome by a study design using well-defined and strictly controlled experimental conditions, as well as intraindividual comparisons. Improvements in the technology of the indirect calorimetry equipment have led to great advances in accuracy and temporal resolution of the outcome parameters, as well as lowering of maintenance costs. New respiratory chambers are currently built in places all over the world, indicating a renaissance of this classical approach. Maintenance and capacity of these unique and rare research facilities with metabolic chambers will benefit from knowledge transfer in translational science (for example, correct adjustment of energy expenditure for changes in body composition in human and animal studies), as well as from an international network of expertise that may facilitate future collaborations in joint applications for consortium projects.

Future studies will progressively integrate traditional state-ofthe-art technology for assessment of energy balance with mechanistic insights provided by molecular sciences and translational research. The results will help to further reveal the truthfulness of myths and presumptions on strategies for prevention and treatment of obesity.

The Symposium on Regulation of Energy Balance held on 10 September at the University of Hohenheim, Germany, brought together international experts in the field and focused on relevant methodological issues regarding the measurement of energy and macronutrient balance, as well as on current topics in obesity research, such as metabolic adaptation to weight loss, the etiology of the catch-up fat phenomenon during weight regain, the carbohydrate-insulin hypothesis of obesity, coldinduced thermogenesis in humans and control of energy expenditure.

\section{CONFLICT OF INTEREST}

The authors declare no conflict of interest.

A Bosy-Westphal University of Hohenheim, Institute for Nutritional Medicine (180c), Stuttgart, Germany

E-mail: Anja.Bosy-Westphal@uni-hohenheim.de 